\title{
CURSO MODELADO DE NICHO ECOLÓGICO, VERSIÓN 1.0
}

A. Townsend Peterson ${ }^{1}$, Robert P. Anderson ${ }^{2}$, Marlon E. Cobos $^{1}$, Martín Cuahutle $^{3}$, Angela P. Cuervo-Robayo ${ }^{3,4}$, Luis E. Escobar ${ }^{5}$, Marc FernandeZ ${ }^{6}$, Daniel Jiménez-García ${ }^{7}$, Andrés Lira-Noriega ${ }^{8}$, Jorge M. Lobo ${ }^{9}$, Fernando Machado-Stredel ${ }^{1}$, Enrique MartínezMeyer $^{4,10}$, Claudia Nuñez-Penichet ${ }^{1}$, Javier Nori ${ }^{11}$, Luis Osorio-Olvera ${ }^{10}$, María Teresa RodrígueZ ${ }^{3}$, Octavio Rojas-Soto ${ }^{8}$, Daniel Romero-Álvarez ${ }^{1}$, Jorge Soberón ${ }^{1}$, Sara Varela ${ }^{12}$, Y CARlos YAÑEZ-ARENAS ${ }^{13}$

${ }^{1}$ Biodiversity Institute, University of Kansas, Lawrence, Kansas 66045 USA; ${ }^{2}$ Department of Biology, City College of New York, City University of New York, 160 Convent Avenue, New York, NY 10031 USA; Doctoral Program in Biology, Graduate Center, City University of New York, 365 5th Avenue, New York, NY 10016 USA; American Museum of Natural History, Central Park West at 79 ${ }^{\text {th }}$ Street, New York, NY 10024 USA; ${ }^{3}$ Comisión Nacional para el Conocimiento y Uso de la Biodiversidad (Conabio), Ciudad de México, C.P., México; ${ }^{4}$ Instituto de Biología, Universidad Nacional Autónoma de México, México City 04510, México; ${ }^{5}$ Department of Fish and Wildlife Conservation, Virginia Tech, Blacksburg, Virginia 24061 USA; ${ }^{6}$ Centre for Ecology, Evolution and Environmental Changes/Azorean Biodiversity Group, and Faculdade de Ciências e Tecnologia, Universidade dos Açores, Ponta Delgada, 9501-801, Portugal; ${ }^{7}$ Centro de Agroecología y Ambiente, Instituto de Ciencias, Benemérita Universidad Autónoma de Puebla. Edif. Val1, Ecocampus-BUAP. Km 1.7 Carretera San Baltazar Tetela, San Pedro Zacachimalpa. C.P. 72960, Puebla, Puebla, México.; ${ }^{8}$ Instituto de Ecología, A.C.; Carretera antigua a Coatepec No. 351, El Haya, 91070 Xalapa, Veracruz, México.; ' Dept. Biogeography and Global Change, National Museum of Natural Sciences, c/ José Gutiérrez Abascal, 2, 288006, Madrid, Spain; ${ }^{10}$ Centro del Cambio Global y la Sustentabilidad en el Sureste AC, CP 86080, Villahermosa, Tabasco, Mexico.; ${ }^{11}$ Instituto de Diversidad y Ecología Animal (IDEA-CONICET) and Centro de Zoología Aplicada, Universidad Nacional de Córdoba,Córdoba, Argentina; ${ }^{12}$ Museum für Naturkunde Leibniz Institut für Evolutions und Biodiversitätsforschung. Invalidenstraße 43. D-10115 Berlin, Germany; ${ }^{13}$ Laboratorio de Ecología Geográfica, Unidad de Biología de la Conservación, Parque Cientifico y Tecnológico de Yucatán, Facultad de Ciencias, Universidad Nacional Autónoma de México, 97302 Sierra Papacal, Yucatán, México.

Resumen.-El conjunto de ideas, métodos y programas informáticos que se conoce como "Modelado de Nicho Ecológico" (MNE) el relacionado "Modelado de Distribución de Especies" (MDS) han sido objeto de intensa exploración e investigación en las últimas décadas. A pesar de existir al menos cuatro síntesis publicadas, este campo ha crecido tanto en complejidad, que la formación de nuevos investigadores es difícil. Hasta ahora, dicha formación se ha hecho de manera presencial en cursos organizados por universidades o centros de investigación, de los que hemos formado parte como instructores. Sin embargo, el acceso a este tipo de cursos especializados es restringido, por un lado, porque los cursos no se ofrecen en todas las universidades, y por otro, porque normalmente se imparten en inglés. Para facilitar el acceso a una mayor comunidad de científicos de habla hispana, presentamos un curso en español, completamente digital y de acceso gratuito, que se realizó vía Internet durante 23 semanas consecutivas en 2018. Aunque las barreras intrínsecas al uso de Internet pueden dificultar la accesibilidad a los materiales del curso, hemos usado diversos formatos para la divulgación de los contenidos académicos (video, audio, pdf) con el objetivo de eliminar la mayor parte de estos problemas.

Abstract.-The suite of ideas, protocols, and software tools that has come to be known as "Ecological Niche Modeling" (ENM) — as well as those for the related "Species Distribution Modeling" (SDM) - has seen intensive exploration and research attention in recent decades. In spite of at least four syntheses, the field has grown so much in complexity that it is rather difficult to access for newcomers. Until now, accessibility to this field was achieved by in-person courses organized by universities or research centers, in some of which we have participated as instructors. However, the access to these 
specialized courses is limited, on one hand because they are not offered in all universities, and on the other because normally they are taught in English. To expand the access to a wider community of Spanish-speaking researchers, here we offer an entirely digital and free-of-charge course in Spanish, which was presented over 23 weeks via Internet in 2018. Although intrinsic Internet-related barriers may limit access to course materials, we have made them available in diverse formats (video, audio, pdf) in order to eliminate most of these problems.

Palabras claves: modelado de nicho ecológico, modelado de distribucion de especies, ecología, biogeografía, curso en línea

Key Words: ecological niche modeling, species distribution modeling, ecology, biogeography, online course

Muchas de las preguntas en ecología y biogeografía giran en torno a la posibilidad de disponer de un conocimiento detallado sobre la distribución de las especies. Esto incluye la ecología poblacional (e.g., qué condiciones necesita una especie para mantener a una población), biogeografía (e.g., qué o cuántas especies se encuentran en diferentes lugares del planeta), conservación (e.g., qué áreas necesitan ser protegidas para asegurar la supervivencia de un grupo de especies) y el estudio de las invasiones biológicas (e.g., qué áreas pueden ser colonizadas por una especie invasora en particular). El gran valor de este tipo de información relacionada con la distribución geográfica de las especies incrementó recientemente debido a la popularidad de un tipo de métodos generados a finales de los años 70 y principios de los 80 (Soto et al. 1984; Nix 1986) y que se han desarrollado intensamente en los últimos 20 años (Peterson et al. 1999; Guisan y Zimmermann 2000; Elith et al. 2006).

La base conceptual de este campo, que podría denominarse "ecología distribucional", está en los trabajos clásicos de ecología y biogeografía (Andrewartha y Birch 1964; Udvardy 1969), pero su sentido moderno fue desarrollado en las últimas dos décadas. Pulliam (2000) desarrolló el primer conjunto de ideas modernas, que luego fue interpretado y reelaborado por Soberón y Peterson (2005). Más tarde se editaron una serie de síntesis extensas (Franklin 2010; Peterson et al. 2011; Peterson 2014; Guisan et al. 2017) al mismo tiempo que se generó una serie de trabajos desarrollando nuevos conceptos o discutiendo los fundamentos del campo (Araújo y Guisan 2006; Jiménez-Valverde et al. 2008; Anderson 2012). Además, numerosos avances metodológicos han sido publicados en forma de artículos científicos, opiniones e ideas sobre diversas elecciones metodológicas (e.g., Radosavljevic y Anderson 2014; Zhu y Peterson 2017; Qiao et al. 2018). Por lo tanto, esta especialidad, la ecología distribucional, se ha convertido en un campo difícil de abarcar, provocando que los estudiantes e investigadores que buscan comenzar su formación en la materia puedan llegar a sentirse abrumados e incluso frustrados. Aunque la mayoría de la literatura científica sobre la ecología distribucional ha sido publicada en inglés, con ciertas excepciones (Mateo et al. 2011; Varela et al. 2014; Anderson 2015; Soberón et al. 2017), investigadores de España y Latinoamérica han sido líderes en el establecimiento y crecimiento del campo. Además, la gran biodiversidad en Latinoamérica también ha contribuido en la demanda de fuentes de entrenamiento en estas técnicas en la región.

Gran parte de nosotros, individual y colectivamente, hemos realizado distintos cursos en el campo, contribuyendo con el establecimiento de los principios básicos del aporte digital que aquí tratamos. A pesar de que dichos cursos han mostrado una alta participación y han sido realizados en diversos países (Argentina, Brasil, Chile, Colombia, Ecuador, España, México, Perú y Venezuela), estas iniciativas presentan limitaciones obvias si se busca alcanzar a la mayoría de los hispanohablantes interesados en el campo. Por lo tanto, elaboramos un curso en línea sobre modelado de nicho, gratuito y en español, durante el período marzo a agosto de 2018. Dicho curso fue dictado por un grupo de 21 instructores que trabajan actualmente en seis países, realizando investigación activa y con amplia experiencia en la ecología distribucional. Ya que esta iniciativa se encuentra en línea en formato de acceso abierto, presentamos esta contribución como un recurso que esperamos sea de utilidad para una comunidad más amplia. Sin dudas, anticipamos que el presente material requerirá ser revisado y actualizado en el futuro, de manera que se mantenga como una herramienta pertinente en el campo de la ecología distribucional. 


\section{El Curso}

El curso fue impartido durante 23 semanas. Fue organizado en los siguientes tópicos generales: introducción al concepto de nicho ecológico, variables ambientales, datos de presencia, visualización, el área de calibración (M), el diagrama BAM (i.e., factores del ambiente abiótico, del ambiente biótico y de movimiento, que afectan distribuciones de especies; sensu Soberón y Peterson 2005), algoritmos, evaluación de modelos, transferencia de modelos, comparaciones de nichos, aplicaciones y conclusiones. El material de cada semana fue presentado en una serie de video conferencias publicadas cada lunes, junto a versiones descargables en distintos formatos, así como una sesión posterior de preguntas y respuestas cada viernes. La Tabla 1 resume el curso y los diferentes elementos incluidos.

Las presentaciones consistieron en grabaciones de audio de los instructores con captura simultánea de diapositivas. Esto aseguró una calidad alta de audio y video, en lugar de instructores filmados frente a una pantalla como en cursos anteriores (Peterson y Ingenloff 2015). Todo el material se encuentra disponible a través de un centro de intercambio de información de enlaces en la página web de Biodiversity Informatics Training Curriculum. Para el final del

Tabla 1. Resumen del curso de Modelado de Nicho Ecológico, Versión 1.0, incluyendo los temas principales, título específico, enlaces para las versiones en formato .pdf, .mp3, y .mp4, así como los enlaces a los videos en YouTube, materiales descargables e instructor para cada tema.

\begin{tabular}{|c|c|c|c|c|c|c|c|}
\hline Unidad & Título & PDF & MP3 & YouTube & MP4 & $\begin{array}{l}\text { Material } \\
\text { adicional }\end{array}$ & Instructor \\
\hline \multirow[t]{7}{*}{ Introducción } & & & & & & & \\
\hline & Plan del Curso & $\underline{\mathrm{PDF}}$ & $\underline{\mathrm{MP3}}$ & $\underline{\mathrm{YT}}$ & $\underline{\text { MP4 }}$ & & A. Townsend Peterson \\
\hline & $\begin{array}{l}\text { Introducción a la ecología de distribuciones } \\
\text { de especies }\end{array}$ & $\underline{\mathrm{PDF}}$ & $\underline{\text { MP3 }}$ & $\underline{\mathrm{YT}}$ & $\underline{\text { MP4 }}$ & & A. Townsend Peterson \\
\hline & Preguntas y respuestas & & & $\underline{\mathrm{YT}}$ & & & Todos \\
\hline & $\begin{array}{l}\text { Elementos de una teoría del nicho } \\
\text { Grinnelliano }\end{array}$ & $\underline{\mathrm{PDF}}$ & $\underline{\text { MP3 }}$ & $\underline{\mathrm{YT}}$ & $\underline{\text { MP4 }}$ & Enlace & Jorge Soberón \\
\hline & Mirando un mapa & $\underline{\mathrm{PDF}}$ & $\underline{\mathrm{MP3}}$ & $\underline{\mathrm{YT}}$ & $\underline{\text { MP4 }}$ & & Jorge M. Lobo \\
\hline & Preguntas y respuestas & & & $\underline{\mathrm{YT}}$ & & & Todos \\
\hline \multirow[t]{11}{*}{$\begin{array}{l}\text { Datos } \\
\text { Ambientales }\end{array}$} & & & & & & & \\
\hline & Relación a teoría de nicho & $\underline{\mathrm{PDF}}$ & $\underline{\text { MP3 }}$ & $\underline{\mathrm{YT}}$ & $\underline{\text { MP4 }}$ & Enlace & Angela Cuervo Robayo \\
\hline & Datos ambientales - práctica & $\underline{\mathrm{PDF}}$ & $\underline{\mathrm{MP3}}$ & $\underline{\mathrm{YT}}$ & $\underline{\text { MP4 }}$ & & Daniel Jiménez García \\
\hline & Preguntas y respuestas & & & $\underline{\mathrm{YT}}$ & & & Todos \\
\hline & Datos de climas, con práctica en $\mathrm{R}$ & $\underline{\mathrm{PDF}}$ & $\underline{\text { MP3 }}$ & $\underline{\mathrm{YT}}$ & $\underline{\text { MP4 }}$ & Enlace & Angela Cuervo Robayo \\
\hline & $\begin{array}{l}\text { AOGCMs y diferencias entre worldclim y } \\
\text { AOGCMs }\end{array}$ & $\underline{\mathrm{PDF}}$ & $\underline{\text { MP3 }}$ & $\underline{\mathrm{YT}}$ & $\underline{\text { MP4 }}$ & & $\underline{\text { Sara Varela }}$ \\
\hline & Datos de ambientes marinos y su dinámica & $\underline{\mathrm{PDF}}$ & $\underline{\text { MP3 }}$ & $\underline{\mathrm{YT}}$ & $\underline{\text { MP4 }}$ & & $\underline{\text { Marc Fernandez }}$ \\
\hline & Preguntas y respuestas & & & $\underline{\mathrm{YT}}$ & & & Todos \\
\hline & Datos de sensores remotos & $\underline{\mathrm{PDF}}$ & $\underline{\text { MP3 }}$ & $\underline{\mathrm{YT}}$ & $\underline{\text { MP4 }}$ & & $\frac{\text { Teresa Rodríguez y }}{\underline{\text { Martín Cuahutle }}}$ \\
\hline & $\begin{array}{l}\text { Procesamiento de datos ambientales: } \\
\text { preparación, reducción, selección }\end{array}$ & $\underline{\mathrm{PDF}}$ & $\underline{\mathrm{MP3}}$ & $\underline{\mathrm{YT}}$ & $\underline{\text { MP4 }}$ & & Claudia Nuñez-Penichet \\
\hline & Preguntas y respuestas & & & $\underline{\mathrm{YT}}$ & & & Todos \\
\hline \multicolumn{8}{|l|}{$\begin{array}{l}\text { Datos de } \\
\text { Presencia }\end{array}$} \\
\hline & $\begin{array}{l}\text { Relación a teoría de nicho ¿Qué son } \\
\text { presencias y qué son ausencias? }\end{array}$ & $\underline{\mathrm{PDF}}$ & $\underline{\text { MP3 }}$ & $\underline{\mathrm{YT}}$ & $\underline{\text { MP4 }}$ & \multirow{2}{*}{$\frac{\text { Simões paper, }}{\text { Saupe paper }}$} & Jorge Soberón \\
\hline & Unidades de modelado & $\underline{\mathrm{PDF}}$ & $\underline{\text { MP3 }}$ & $\underline{\mathrm{YT}}$ & $\underline{\text { MP4 }}$ & & Octavio Rojas Soto \\
\hline & Preguntas y respuestas & & & $\underline{\mathrm{YT}}$ & & & Todos \\
\hline & Fuentes de datos & $\underline{\mathrm{PDF}}$ & $\underline{\mathrm{MP3}}$ & $\underline{\mathrm{YT}}$ & $\underline{\text { MP4 }}$ & & A. Townsend Peterson \\
\hline & Georeferenciación & $\underline{\mathrm{PDF}}$ & $\underline{\text { MP3 }}$ & $\underline{\mathrm{YT}}$ & $\underline{\text { MP4 }}$ & Enlace & Daniel Jiménez García \\
\hline & Preguntas y respuestas & & & $\underline{\mathrm{YT}}$ & & Enlace & Todos \\
\hline & Control de calidad y reducción & $\underline{\mathrm{PDF}}$ & $\underline{\mathrm{MP3}}$ & $\underline{\mathrm{YT}}$ & $\underline{\text { MP4 }}$ & Enlace & $\frac{\text { Fernando Machado- }}{\text { Stredel }}$ \\
\hline & Subconjuntos para evaluación & $\underline{\mathrm{PDF}}$ & $\underline{\text { MP3 }}$ & $\underline{\mathrm{YT}}$ & $\underline{\text { MP4 }}$ & Enlace & A. Townsend Peterson \\
\hline & Preguntas y respuestas & & & $\underline{\mathrm{YT}}$ & & & Todos \\
\hline
\end{tabular}


Visualización

Visualización de los datos ambientales Intro a NicheA

NicheA: Práctica

Preguntas y respuestas

M y Configuración BAM

Relación a teoría de nicho
Estimados de $\mathbf{M}$
Limitantes en que se puede modelar y
que no
Preguntas y respuestas

Algorithmos

Relación a teoría de nicho

"The Good, The Bad, and the Ugly," "Un Solo Dios" y balas de plata Preguntas y respuestas Incertidumbre: introducción Incertidumbre: conceptos básicos Evaluación de incertidumbre

Selección de modelos, y control de sobreajuste en calibración Preguntas y respuestas

Evaluación

Relación a teoría de nicho

Datos para evaluación

Probabilidad, favorabilidad e idoneidad

Preguntas y respuestas

Validación, discriminación y calibración: ¿Cómo podemos validar un modelo? Evaluación no dependiente de un umbral (ROC y ROC parcial)

ROC parcial, teoría y práctica.

Preguntas y respuestas

Más sobre evaluación: umbrales, pruebas dependientes de umbral, rendimiento, etc.

Preguntas y respuestas

Transferencia

Transferencias 1

Transferencias 2

Pasado, presente, futuro

Preguntas y respuestas

Owens et al. - MOP y extrapolación

Implementación de MOP

Preguntas y respuestas

Comparación

Relación a teoría de nicho

$\begin{array}{lccc}\frac{\mathrm{PDF}}{\mathrm{PDF}} \frac{\mathrm{MP}}{\mathrm{MP3}} & \frac{\mathrm{YT}}{\mathrm{YT}} & \frac{\text { MP4 }}{\mathrm{MP4}} & \underline{\underline{\text { Datos }}} \\ \underline{\mathrm{PDF}} \underline{\mathrm{MP3}} & \frac{\underline{\mathrm{YT}}}{\underline{\mathrm{YT}}} & \underline{\text { MP4 }} & \underline{\underline{\text { Datos }}} \\ \text { GeoDa, Enlace }\end{array}$

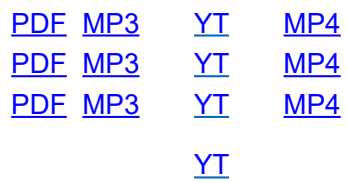
$\underline{\mathrm{PDF}} \underline{\mathrm{MP} 3} \quad \underline{\mathrm{YT}} \quad \underline{\mathrm{MP}} 4$

$\underline{\mathrm{PDF}} \underline{\mathrm{MP} 3} \quad \underline{\mathrm{YT}} \quad \underline{\mathrm{YT}} \quad \underline{\mathrm{MP}} 4$

$\underline{\mathrm{PDF}} \underline{\mathrm{MP} 3} \quad \underline{\mathrm{YT}} \quad \underline{\mathrm{MP}} 4$

$\underline{\mathrm{PDF}} \underline{\mathrm{MP} 3} \quad \underline{\mathrm{YT}} \quad \underline{\mathrm{MP}} 4$

$\underline{\mathrm{PDF}} \underline{\mathrm{MP} 3} \quad \underline{\mathrm{YT}} \quad \underline{\mathrm{MP}} 4$

$\underline{\mathrm{YT}}$

Ebola, climate
Daniel Jiménez García

Luis Escobar

Luis Escobar
$\underline{\mathrm{PDF}} \underline{\mathrm{MP} 3} \quad \underline{\mathrm{YT}} \quad \mathrm{MP4}$

$\underline{\mathrm{PDF}}$ MP3 $\quad \underline{\mathrm{YT}} \quad \underline{\mathrm{MP}} 4$

$\underline{\mathrm{PDF}}$ MP3 $\quad \underline{\mathrm{YT}} \quad \underline{\mathrm{MP}}$

$\underline{\text { PDF }} \underline{\text { MP3 }} \quad$ YT $\quad$ MP4

\section{Enlace \\ Enlace \\ Enlace \\ Enlace

\author{
Carlos Yañez Arenas \\ A. Townsend Peterson \\ A. Townsend Peterson \\ Todos
}

Jorge Soberón

A. Townsend Peterson

Todos

A. Townsend Peterson

Enlace Enrique Martínez-Meyer

Marlon Cobos

Robert Anderson models

Todos

A. Townsend Peterson

A. Townsend Peterson Jorge M. Lobo

Todos

Jorge M. Lobo

A. Townsend Peterson

Luis Osorio Olvera

Todos

A. Townsend Peterson

Todos

\begin{tabular}{|c|c|c|c|c|}
\hline$\underline{\text { PDF MP3 }}$ & $\underline{\mathrm{YT}}$ & MP4 & Enlace & A. Townsend Peterson \\
\hline$\underline{\text { PDF MP3 }}$ & $\underline{\mathrm{YT}}$ & $\underline{\text { MP4 }}$ & & $\underline{\text { Robert Anderson }}$ \\
\hline$\underline{\text { PDF MP3 }}$ & $\underline{\mathrm{YT}}$ & MP4 & & Enrique Martínez-Meyer \\
\hline & $\underline{\mathrm{YT}}$ & & Enlace & Todos \\
\hline$\underline{\text { PDF MP3 }}$ & $\underline{\mathrm{YT}}$ & MP4 & Enlace & A. Townsend Peterson \\
\hline $\mathrm{DF} \underline{\mathrm{MP} 3}$ & $\underline{\mathrm{YT}}$ & MP4 & & Luis Osorio Olvera \\
\hline
\end{tabular}

Enlace

A. Townsend Peterson

Luis Escobar

Luis Escobar

Enlace Todos 


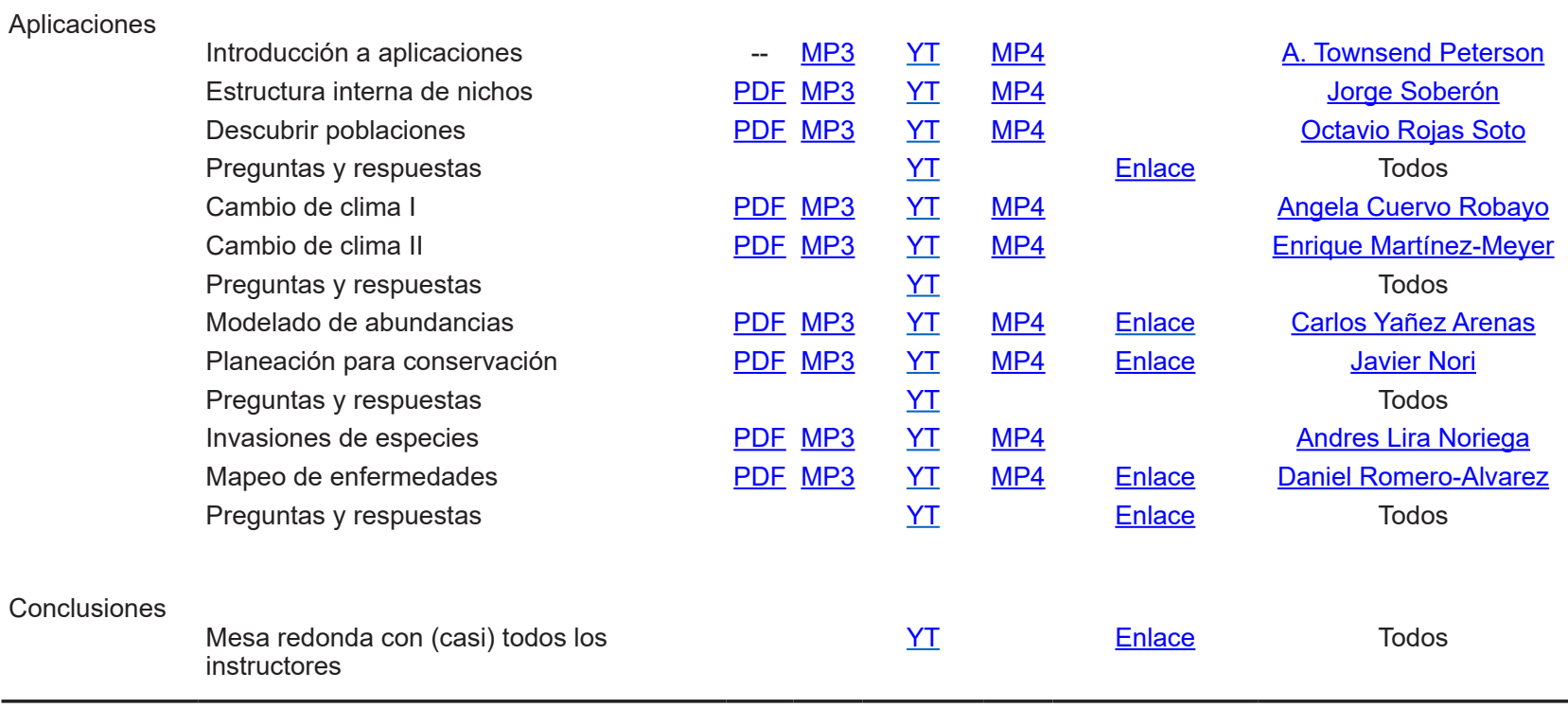

curso, los videos fueron vistos en YouTube un aproximado de 64,000 veces, con un tiempo total de más de 12,500 horas de reproducción. Este formato también permitió que los materiales del curso estuviesen disponibles para descarga directa como archivos de video (.mp4) y audio (.mp3) acompañados de las presentaciones utilizadas (.pdf; Curso Modelado de Nicho Ecológico 2018). Anticipamos que debido a la diversidad de espectadores del curso, diferentes tipos de acceso iban a ser considerados como óptimos, por lo cual usamos diversos recursos digitales. En una encuesta de recapitulación del curso, con 354 participantes, 50.0\% reportaron usar videos de YouTube como su modo de acceso, 19.2\% descargaron archivos .mp4 y $2.2 \%$ usaron archivos .mp3. El 28.5\% de los participantes descargó las diapositivas en formato .pdf como auxiliar a las presentaciones en archivo de audio o video.

Todos los estudiantes tuvieron la oportunidad de enviar preguntas a los instructores a través de un Formulario de Google, los instructores pudieron responder una porción de estas preguntas - las más comunes - al final de cada semana. A pesar de no ser respondidas en su totalidad, las preguntas fueron una manera útil de verificar la participación (i.e., participantes que enviaron preguntas más de 11 semanas) y también para averiguar lo entendido o parcialmente asimilado por la audiencia en las presentaciones (Fig. 1). Al final del curso contamos aproximadamente 6,200 preguntas; un patrón consistente en la inquietud de los participantes del curso fue la selección de las variables ambientales necesarias para calibrar los modelos de nicho. Las preguntas frecuentes fueron: Cuáles variables usar? y Cómo seleccionarlas?
¿Cuántas usar? Además, un tema de interés común fue la evaluación de la transferencia de modelos hacia otras regiones o períodos, que son aplicaciones de estas herramientas para evaluar zonas de riesgo para especies invasoras y potenciales efectos del cambio climático, respectivamente. Otro tema particularmente dominante en las preguntas de los participantes, fue sobre la selección del algoritmo ideal para utilizar en los distintos estudios (Fig. 1).

Hasta donde sabemos, este material curricular representa el conjunto más exhaustivo de recursos de entrenamiento para el campo disponible en cualquier idioma. El grupo de instructores representó diversas

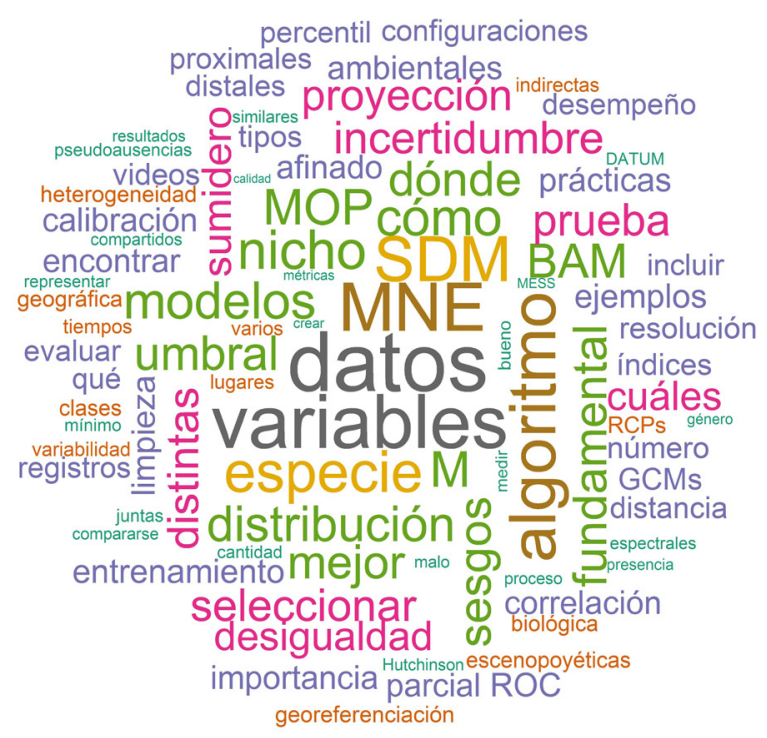

Figura 1. Palabras más frecuentes encontradas en las preguntas de los participantes del curso de Modelado de Nicho Ecológico. 
áreas del conocimiento de la ecología distribucional, permitiendo abarcar una diversidad considerable de temas y herramientas. Consideramos que el curso fue efectivo, con base en la diversidad de países representados por instructores y participantes, el alto número de participantes y su compromiso al atender a la mayoría de las sesiones semanales. Así, proponemos que esta estructura de educación puede ser empleada por otras disciplinas para facilitar la educación de acceso libre y a distancia, facilitando la trasferencia de tecnología e información hacia (y entre) países en desarrollo. Por su forma modular, este curso se puede actualizar y evolucionar, según los intereses y deseos de los miembros de la comunidad. Los videos de las presentaciones son accesibles en internet de forma gratuita, y pueden ser actualizados y mejorados a través del tiempo; además, esperamos la participación de la comunidad global para la traducción de los materiales generados en este curso a otros idiomas.

\section{AgradeCimiEnTos}

Agradecemos al resto de los miembros del Grupo de Modelado de Nicho Ecológico de la Universidad de Kansas por su aporte y asistencia. Las presentes contribuciones representan difusión científica por parte de varias instituciones y agencias de financiamiento (incluyendo U.S. NSF DBI-1661510 para R.P. Anderson y Virginia Tech Startup Funds para L.E. Escobar).

\section{Literature Cited}

Anderson, R. P. 2012. Harnessing the world's biodiversity data: Promise and peril in ecological niche modeling of species distributions. Annals of the New York Academy of Sciences 1260:66-80.

Anderson, R. P. 2015. El modelado de nichos y distribuciones: No es simplemente "clic, clic, clic". Biogeografía 8:4-27.

Andrewartha, H. G., and L. C. Birch. 1964. The Distribution and Abundance of Animals. University of Chicago Press, Chicago.

Araújo, M. B., and A. Guisan. 2006. Five (or so) challenges for species distribution modelling. Journal of Biogeography 33:1677-1688.

Elith, J., C. H. Graham, R. P. Anderson, M. Dudik, S. Ferrier, A. Guisan, R. J. Hijmans, F. Huettmann, J. R. Leathwick, A. Lehmann, J. Li, L. G. Lohmann, B. A. Loiselle, G. Manion, C. Moritz, M. Nakamura, Y. Nakazawa, J. M. Overton, A. T. Peterson, S. J. Phillips, K. Richardson, R. Scachetti-Pereira, R. E. Scha- pire, J. Soberon, S. Williams, M. S. Wisz, and N. E. Zimmermann. 2006. Novel methods improve prediction of species' distributions from occurrence data. Ecography 29:129-151.

Franklin, J. 2010. Mapping Species Distributions: Spatial Inference and Prediction. Cambridge University Press, Cambridge.

Guisan, A., W. Thuiller, and N. E. Zimmermann. 2017. Habitat Suitability and Distribution Models: with Applications in R. Cambridge University Press, Cambridge.

Guisan, A., and N. Zimmermann. 2000. Predictive habitat distribution models in ecology. Ecological Modelling 135:147-186.

Jiménez-Valverde, A., J. M. Lobo, and J. Hortal. 2008. Not as good as they seem: The importance of concepts in species distribution modelling. Diversity and Distributions 14:885-890.

Mateo, R. G., Á. M. Felicísimo, and J. Muñoz. 2011. Modelos de distribución de especies: Una revisión sintética. Revista Chilena de Historia Natural 84:217-240.

Nix, H. A. 1986. A biogeographic analysis of Australian elapid snakes. Pp. 4-15 in R. Longmore, ed. Atlas of elapid snakes of Australia. Australian Government Publishing Service, Canberra.

Peterson, A. T. 2014. Mapping Disease Transmission Risk. Johns Hopkins University Press, Baltimore.

Peterson, A. T., and K. Ingenloff. 2015. Biodiversity Informatics Training Curriculum, version 1.2. Biodiversity Informatics 10:65-74.

Peterson, A. T., J. Soberón, R. G. Pearson, R. P. Anderson, E. Martínez-Meyer, M. Nakamura, and M. B. Araújo. 2011. Ecological Niches and Geographic Distributions. Princeton University Press, Princeton.

Peterson, A. T., J. Soberón, and V. Sánchez-Cordero. 1999. Conservatism of ecological niches in evolutionary time. Science 285:1265-1267.

Pulliam, H. R. 2000. On the relationship between niche and distribution. Ecology Letters 3:349-361.

Qiao, H., X. Feng, L. E. Escobar, A. T. Peterson, J. Soberón, G. Zhu, and M. Papeş. 2018. An evaluation of transferability of ecological niche models. Ecography 42:521-534.

Radosavljevic, A., and R. P. Anderson. 2014. Making better Maxent models of species distributions: Complexity, overfitting and evaluation. Journal of Biogeography 41:629-643.

Soberón, J., L. Osorio-Olvera, and A. T. Peterson. 2017. Diferencias conceptuales entre modelación de nichos y modelación de áreas de distribución. Revista Mexicana de Biodiversidad 88:437-441. 
Soberón, J., and A. T. Peterson. 2005. Interpretation of models of fundamental ecological niches and species' distributional areas. Biodiversity Informatics 2:1-10.

Soto, M., M. J. Angulo, O. L. Garduño, and M. Hernández. 1984. Bioclimatología y computación interactiva. Ciencia y Desarrollo 59:153-161.

Udvardy, M. D. F. 1969. Dynamic Zoogeography. Van Nostrand Reinhold Company, New York.
Varela, S., R. G. Mateo, R. García-Valdés, and F. Fernández-González. 2014. Macroecología y ecoinformática: Sesgos, errores y predicciones en el modelado de distribuciones. Revista Ecosistemas 23:46-53.

Zhu, G.-P., and A. T. Peterson. 2017. Do consensus models outperform individual models? Transferability evaluations of diverse modeling approaches for an invasive moth. Biological Invasions 19:2519-2532. 